\title{
SUPERCONDUCTING RADIO-FREQUENCY MODULES TEST FACILITY OPERATING EXPERIENCE
}

\author{
W. Soyars, R. Bossert, C. Darve, B. Degraff, A. Klebaner, A. Martinez, \\ L. Pei, J. Theilacker \\ Fermi National Accelerator Laboratory \\ Batavia, IL, 60510, USA
}

\begin{abstract}
Fermilab is heavily engaged and making strong technical contributions to the superconducting radio-frequency research and development program (SRF R\&D). Four major SRF test areas are being constructed to enable vertical and horizontal cavity testing, as well as cryomodule testing. The existing Fermilab cryogenic infrastructure has been modified to service Fermilab SRF R\&D needs. The first stage of the project has been successfully completed, which allows for distribution of cryogens for a single cavity cryomodule using the existing Cryogenic Test Facility (CTF) that houses three Tevatron satellite refrigerators. The cooling capacity available for cryomodule testing at MDB results from the liquefaction capacity of the CTF cryogenic system. The cryogenic system for a single 9-cell cryomodule is currently operational. The paper describes the status, challenges and operational experience of the initial phase of the project.
\end{abstract}

KEYWORDS: Test facilities, Superconducting RF, ILC, Cryomodule.

\section{INTRODUCTION}

Fermilab is continuing an R\&D program on Superconducting Radio Frequency (SRF) elliptical and spoke cavities for use in future accelerators such as the International Linear Collider (ILC) and High Intensity Neutrino Source (HINS). These potential projects place increasing demands on the performance of SRF components, and on the ability to produce them in large quantities at lower costs. The existing Fermilab cryogenic infrastructure has been modified to service Fermilab SRF R\&D needs [1]. Four major SRF test areas are being constructed to enable cavity and cryomodule testing. The testing is conducted at the ILCTA_MDB, which is an experimental area constructed inside the Meson Detector Building at Fermilab. Liquid nitrogen and liquid helium are supplied to the Meson Detector Building (ILCTA_MDB) through vacuum insulated transfer lines from the Cryogenic Test Facility (CTF). Cryogens are then distributed to 


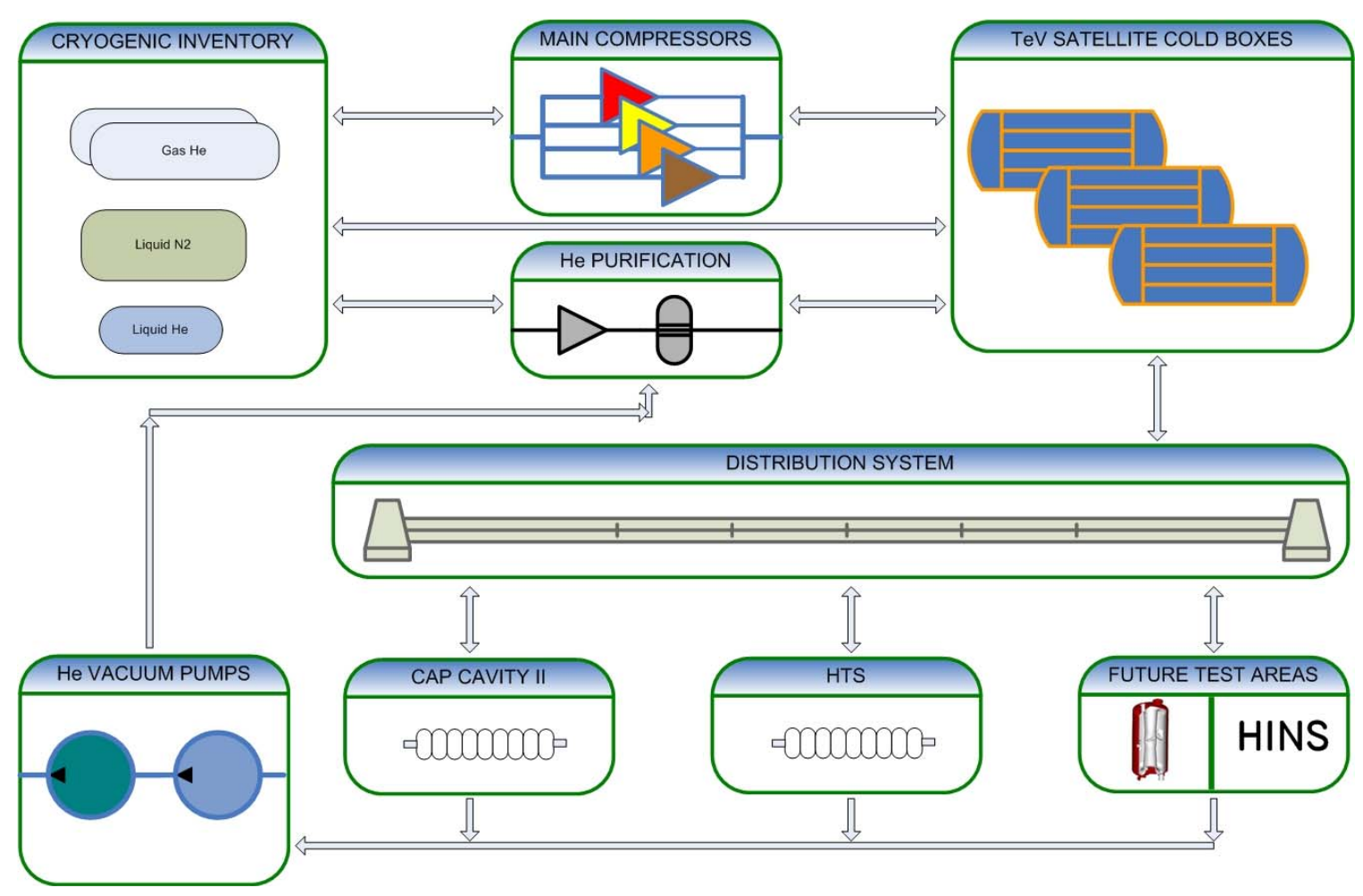

FIGURE 1. The ILCTA_MDB Cryogenic System.

the appropriate test area. A vacuum pump and recovery system allows for closed-loop superfluid Helium production, see FIGURE 1.

Test areas in this facility are divided into the following caves - Capture Cavity II (CC2), Horizontal Test System (HTS), Spoke Resonators Test Cryostat (SRTC), and a front end for the High Intensity Neutrino Source (HINS). The CC2 program has been operational for over a year. Note, CC2 operations at MDB is only temporary; it will be moved and installed in a future beam line. The HTC cryogenic system is also complete. The SRTC and HINS are future programs. All test caves can operate down to $1.6 \mathrm{~K}$, with the exception of the HINS low energy front end which operates in the $4.5 \mathrm{~K}$ range. All test caves could operate simultaneously depending on the operating requirements for specific tests.

\section{CRYOGENIC INFRASTRUCTURE}

\section{Refrigerator Plant}

The ILCTA_MDB cryogenic system consists of compressors, cold boxes, transfer line, and on-line purification system with a dedicated compressor. The majority of these components where originally installed around 1980. The complete transfer line from refrigerator plant location to the MDB test location was first cooled down in September 2005. The cryogenic system has operated with excellent reliability, not contributing any significant downtime to the physics experiments.

Initial operation during the first year used only one refrigerator cold box, which gives a reasonable steady-state liquefier capacity previously defined as about 3-3.5 g/s [1]. For expansion of future loads and to support more frequent load cool-downs, we needed to confirm and understand the control of additional capacity when running two 
refrigerator cold boxes. The third and final cold box is envisioned as always being a spare. A test was conducted in which the output of two cold boxes was delivered to MDB, where a $4.3 \mathrm{~K}$ liquefier load was extracted and metered. For tuning simplicity, the cold boxes were initially configured with one purely operating as a liquefier. However, best output was achieved when we ran two cold-boxes in "mixed mode", with a partial liquefier load on each and cold box temperatures balanced by two-phase return valve regulation. We demonstrated an output of about $6 \mathrm{~g} / \mathrm{s}$ with $200 \mathrm{~W}$ of artificial refrigerator load for plant margin and enhanced stability. This demonstrated that two cold boxes can be tuned with existing hardware to approximately double the one cold box liquefaction rate, as theoretically expected.

\section{Vacuum Pump}

SRF cavities are submerged in a bath of saturated superfluid helium (helium II), conditions which can be achieved using a large capacity standard rotary vacuum pump handling very low pressure helium gas at room temperature. The saturated helium II bath is replenished continuously by a flow of liquid helium II obtained from Joule-Thomson (JT) expansion and phase separation, while the excess vapor is removed by a vacuum pump to lower the SRF cavity bath pressure below $4 \mathrm{kPa}$ (30 torr).

The ILCTA_MDB vacuum pump is designed to achieve up to $10 \mathrm{~g} / \mathrm{s}$ of superfluid helium for the various SRF users. The current nominal configuration calls for a Helium II flow of about $0.5 \mathrm{~g} / \mathrm{s}$ simultaneously to the CC2 and the HTC. Throttling valves and the booster variable frequency drive permit regulation of the vacuum system. The subatmospheric helium vapor is pumped through a $20 \mathrm{~cm}$ header to the vacuum pump, which is located 110 meters away from the SRF experiments. Then the helium is recovered at $110 \mathrm{kPa}$ from the vacuum skid discharge through a $10 \mathrm{~cm}$ header to the suction of the CTF purifier compressor.

The vacuum skid is composed of a booster (Kinney KMBD10000), a liquid ring pump (Kinney KLRC 2100), an oil pump, and appropriate water / oil heat exchangers. The liquid ring pump is operated using a $149 \mathrm{kWatt}$ (200 HP) motor and the booster using a $75 \mathrm{kWatt}(100 \mathrm{HP})$ variable frequency drive motor. An average of 8.5 liter per second (135 gpm) of cooling water is circulating through the heat exchangers to extract approximately $73 \mathrm{kWatt}$ and $146 \mathrm{kWatt}$ from the booster and liquid ring pump, respectively. About two hundred variables monitor and control the vacuum skid. In complement to manual valves, these variables include control valves, pressures, temperatures, level, flow, intensity and vibration read-back, which are measured by means of transmitters, switches and visual gages.

This vacuum skid was originally designed and fabricated by Jefferson Laboratory and Kinney Pump in 1993 before it was refurbished at the Fermilab/Proton Assembly Building for service at ILCTA_MDB. The main challenge is to allow the vacuum pump to operate at sub-atmospheric conditions without carrying over contamination to the CTF compressor system. Indeed, the original vacuum pump components (liquid ring pump and booster) are designed for air flow. To protect the liquid helium refrigeration system from leaks to the sub-atmospheric helium, several upgrades have been carried out. For instance, a sophisticated helium guard system and oil reclamation system have been optimized during the first year of the vacuum pump operation. Dynamic shaft/seal of the booster pump and liquid ring pump are double sealed and were covered with helium guards. Instrumentation feed-throughs feature welded pipe, VCO connectors instead of compression fittings, and vacuum rated flanges. The nitrogen contamination level, 
monitored using a Tevatron-style arc-cell developed at Fermilab, typically averages less than 2 ppm returning from the vacuum pump.

The vacuum skid has been optimized to operate safely with sub-atmospheric helium condition. Since the density of helium is seven times lighter than that of the air, oil is injected at the inlet of the booster pump to allow proper lubrication of its impeller. Anti-cavitation valves were implemented to allow the regulation of the booster inlet pressure. A vibration switch is installed on the vacuum skid frame to monitor excessive vibration.

Three different safety levels are used to protect the vacuum skid. First, the vacuum pump system is equipped with mechanical safety devices like pressure relief valves and spring-loaded parallel plates. Next, The PLC is composed of switches, which will cause the liquid ring pump and the booster to stop if the selected pressures, temperatures, liquid levels or/and vibration exceed predefined thresholds. Finally similar hardwired devices with less stringent thresholds trip the vacuum skid (liquid ring pump and/or booster) to insure the ultimate protection of the vacuum pump.

An automated sequence handles pump start-up and stop. Manual operation is also permitted. Further details regarding the vacuum skid control are described elsewhere [2].

During the initial startup, we experienced a significant weld crack on a booster inlet instrumentation flange that lead to air contamination which saturated the on-line adsorber. A second, separate, and much smaller weld crack occurred after about one month of operation. In this case, the adsorber was able to handle the small amount of air in-leak, and physics operations continued as scheduled for six more weeks, after which the pump was shut down and weld repairs made. There were frequent booster trips on high interstage temperature, due to insufficient cooling oil injection. Increasing oil injection manually by experience addressed this. Excessive vibrations were noticed in the liquid ring pump motor, which was a result of a bad bearing and was replaced. It is likely that the bearing failed due to lack of use for multiple years. Once these commissioning issues where addressed, the pump has operated very reliably.

\section{Purification System}

Although considerable effort was put into assuring that the sub-atmospheric portion of the cryogenic system is leak tight, a purifier compressor was integrated into the discharge side of the vacuum pump in order to boost the discharge pressure of the vacuum pump and to send it through an in-line helium purifier to remove any impurities in the helium stream prior to being reclaimed by the refrigeration system.

The purifier compressor is a Mycom Model 1612C two stage oil flooded screw compressor with a maximum mass flow rate of $30 \mathrm{~g} / \mathrm{s}$. This particular compressor was taken from a previously decommissioned refrigeration system. The compressor consists of a compressor skid as well as the associated oil removal system. The compressor has roughly twice the capacity of the Kinney vacuum pump and therefore in order to match flow rates three different regulation schemes were devised. The first is simply to run the compressor at reduced capacity, maintaining compressor suction by matching the vacuum pump output. The second is to use a bypass valve which bypasses discharge flow of the compressor back to suction. The third method is to supplement the vacuum pump flow with flow from the main compressor suction allowing the purifier compressor to run fully loaded. To prevent possibly contaminated flow from the vacuum pump from entering the main compressor suction, a check valve is installed to the main compressor suction. This third option is the one currently employed since it allows the excess capacity to be used 
by the main compressor system and also allows the compressor to be run at the more efficient fully loaded condition.

The in-line purifier is an existing $60 \mathrm{~g} / \mathrm{s}$ helium purifier originally installed at CTF for initial system purification. In the new configuration, the purifier will be operated continuously during vacuum pump operation. In order to improve the reliability of the purifier, the liquid nitrogen supply transfer line was modified by extending the vacuum jacket to the purifier and increasing the supply valve bullet size. The previous design had a portion of the transfer line uninsulated which made it prone to vapor lock, restricting flow to the purifier.

\section{EXPERIMENTAL AREAS}

\section{Capture Cavity II}

The first SRF accelerator device tested at ILCTA_MDB is Capture Cavity II (CC2) [3], a high gradient $1.3 \mathrm{GHz}$ 9-cell superconducting RF cavity installed into an existing cryomodule [4]. This is an upgraded version of the single nine-cell cryomodule (Capture Cavity I) currently operating at the Fermilab-NICADD Photoinjector Laboratory (FNPL) [5] and will be used in the future to increase the beam energy of this accelerator. Its cryogenic system is shown schematically in FIGURE 2. Initial CC2 cooldown was in February 2006 and it has reliably operated at ILCTA_MDB as required at its design temperature of $1.8 \mathrm{~K}$ to present.

A great deal of ILCTA_MDB radio-frequency operating experience has been gained from CC2 [3, 6-7]. A peak accelerating gradient of $31.3 \mathrm{MV} / \mathrm{m}$ was achieved. Dynamic RF studies were conducted in which a heater was used to maintain steady state conditions with and without RF power. At $30 \mathrm{MV} / \mathrm{m}, 1 \mathrm{~Hz}$ repetition, and $800 \mathrm{msec}$ flattop, a dynamic load of $0.17+/-0.03 \mathrm{~W}$ was observed, which translates to a cavity $\mathrm{Q}_{0}=1.5 \mathrm{e} 10$ [8]. Mechanical vibration studies have also been conducted [9].

Cryogenic performance observations have been gained. An automated pumpdown sequence from $4.3 \mathrm{~K}$ to $1.8 \mathrm{~K}$ takes about 45 minutes to achieve steady-state, with a superfluid pressure stability of $+/-13 \mathrm{~Pa}$, see FIGURE 3 . A $4 \mathrm{~W}$ static heat load to $2 \mathrm{~K}$ has also been measured. By applying an artificial heat load to the superfluid helium bath, we demonstrated that the cryogenic system is capable of handling a dynamic component of up to $8 \mathrm{~W}$ at $1.8 \mathrm{~K}$, limited by pressure drop to the vacuum pump. The CC2 feedcan has a small, shell-and-tube, heat exchanger to subcool single phase liquid He from the transfer line. This exchanger is observed to have a steady-state thermal effectiveness of $66 \%+/-4 \%$ with about 27-54 Pa pressure drop at typical operating conditions.

The cryogenic system did produce an unwanted influence on SRF cavity vibrations, when a $20 \mathrm{~Hz}$ mechanical vibration was observed in the cavity, preventing stable RF operations. The vibrations occur when the LHe supply pressure is $>250 \mathrm{kPa}$ and disappear when pressure is lowered, leading to the strong hypothesis that the vibrations are flow induced. In the future, we plan on making direct vibration readings of the supply valve stem and the return pumping line to test this hypothesis. Pressure is now controlled for a nominal supply pressure of $210 \mathrm{kPa}$.

One technical development guided by CC2 experience has been to find a rupture disk the offers superior leak-tight performance under continuous vacuum. This is important because Fermilab standard requirements mandate an ASME-code style primary relief piped directly to the cold, low pressure side of cavity. The best relief leak rate we 


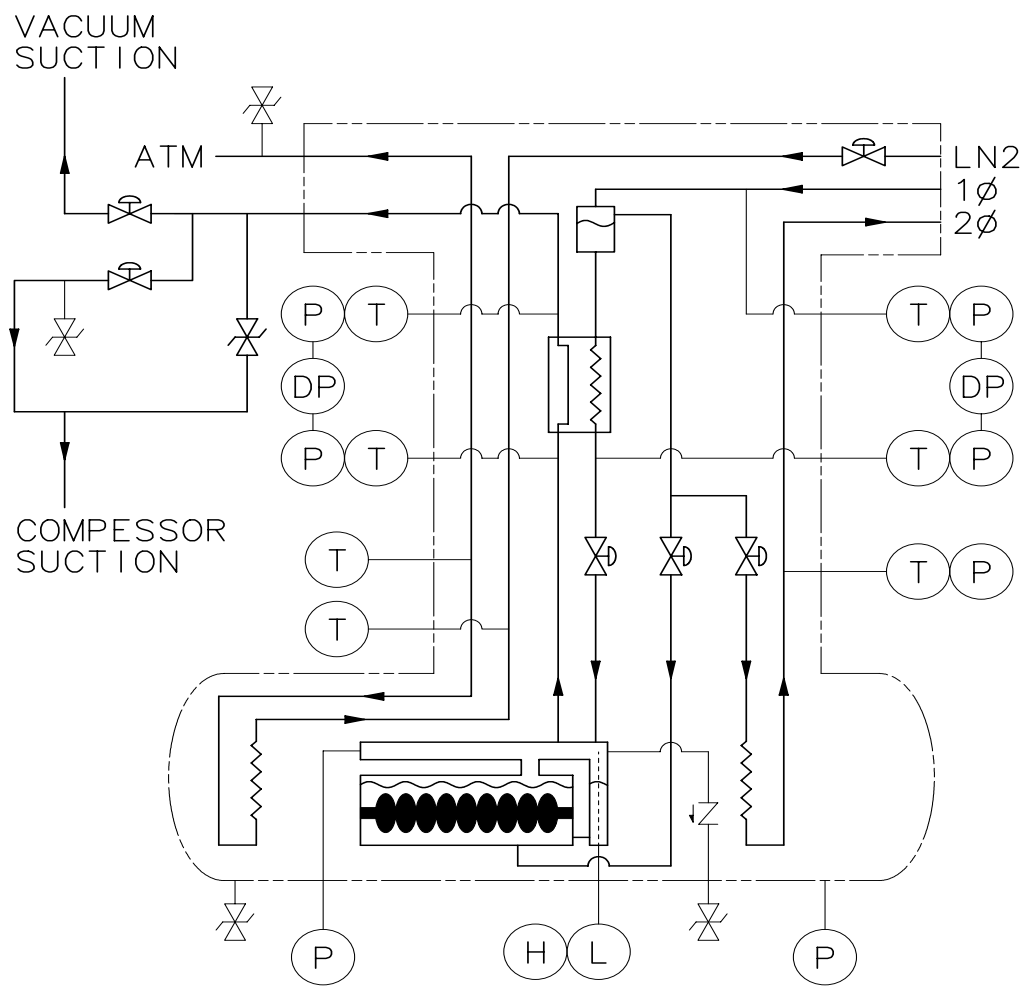

FIGURE 2. Capture Cavity II cryogenic system schematic.

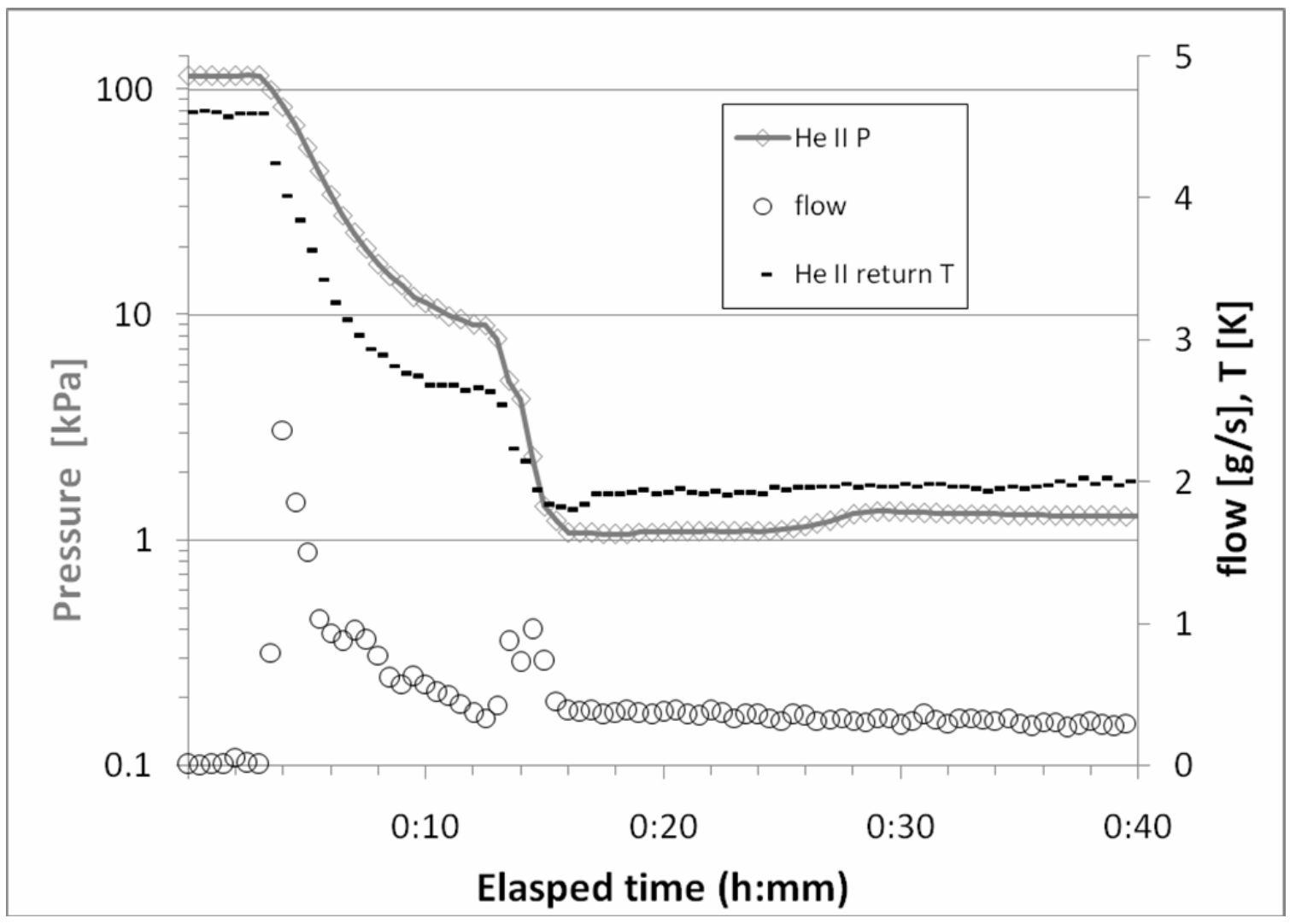

FIGURE 3. Capture Cavity II pump-down to $1.74 \mathrm{~K}$ (1330 Pa). 
currently have tested gives about $10^{-7} \mathrm{~cm}^{3} / \mathrm{sec}$ He leak rate, and we plan on working with vendors to pursue improvement upon this.

\section{Future Test Areas}

The Horizontal Test System (HTS) is designed for dressed cavities to be tested with high pulsed RF power. The purpose of these horizontal tests is to verify that the cavities can achieve the desired accelerating gradient and $Q_{0}$ in order to qualify them for assembly into a cryomodule. Such tests are currently required to separate effects associated with cavity dressing from those associated with cryomodule assembly.

The HTS is comprised of a cryogenic distribution system and Horizontal Test Cryostat (HTC). The HTC resides in a shielding cave in the Meson Detector Building designed to contain X-ray radiation from the cavities. The cryostat design is based on DESY CHECHIA. It allows for dressed cavities to be installed without a need for multilayered insulation.

The HTS was successfully commissioned in April 2007. During the commissioning phase it was proven that the HTS is capable of operating simultaneously with other test areas at ILCTA_MDB. We were able to both cool-down and warm-up the HTC, while operating Capture Cavity II at $1.8 \mathrm{~K}$.

Other future test areas at MDB will be dedicated to the High Intensity Neutrino Source (HINS). For HINS R\&D, a cave located adjacent to the HTS is configured for 325 $\mathrm{MHz}$ SRF applications. An individual cavity (spoke resonator) and a production coupler will be tested in a dedicated cryostat. Once the design of the spoke resonator is established, all the cavities will be tested before they are installed in cryo-modules. After Capture Cavity II testing is concluded and it is relocated into its beamline, plans call for the front end of the HINS to be assembled in the allocated MDB area. The HINS front end will feature an ion source, debuncher cavities, an RF quadrupole, warm spoke resonators and superconducting solenoids, followed by the series of cryomodules, including the spoke resonators.

\section{CONCLUSIONS}

The cryogenic infrastructure to support Fermilab's SRF R\&D program at ILCTA_MDB Test Area is successfully operating. For over a year, reliable closed-loop production of superfluid helium has occurred for SRF operations. Experience with the refrigerators, modified vacuum pump, and on-line purification system has been presented. The commissioning and operation of this cryogenic system is an important step for dependable and cost effective cavity and cryomodule testing at Fermilab.

The accelerator component tested during this period has been the Capture Cavity II 9-cell cavity cryomodule. This testing has enabled Fermilab to gain important SRF operating experience. The cryogenic performance of the Capture Cavity II has been studied. Topics include valve regulation, pump-down sequence, static and dynamic heat loads, vibration effects, heat exchanger effectiveness, and hardware worthiness. The ILCTA_MDB cryogenic system is ready to support future SRF efforts at the Horizontal Test System and other test areas. 


\section{ACKNOWLEDGEMENTS}

This work is supported by the U. S. Department of Energy under contract No. DE-AC02-07CH11359. The authors wish to recognize the dedication and skills of the Accelerator Cryogenics Department technical personnel involved in the installation and operation of this system: Ken Olesen, Greg Johnson, Terry Cross, Roger Milholland, Steve Cozzens, Mark Nylund, Joe Brown, Mike Dinnon, John Juneau, Rich Michals, Shelly Hentges, Ed Altpeter, Wilson Cross, Jeff Spencer, Tim DeGraff, Paul Forester, Bruce Wilson, Ted Roberts, Fannie Linton, Wayne Johnson, John Thompson, Bill Martin, Jim O’Neill, Mike Cooper and Frank Rucinski. We also express thanks for the innovative work performed by Cary Kendziora's team for the modifications of the vacuum skid, with special credit to Mark Ruschman. Gary Ganster made significant contributions to the efficient and reliable controls interface.

\section{REFERENCES}

1. Klebaner, A. L. and Theilacker, J. C., “Cryogenics for the Superconducting Module Test Facility,” in Advances in Cryogenic Engineering 51B, edited by J. G. Weisend, American Institute of Physics, Melville, New York, 2006, pp. 1428-1435.

2. Norris, B. et. al, “Cryogenic Control's for Fermilab’s SRF Cavities and Test Facility,” submitted to Advances in Cryogenic Engineering 53, American Institute of Physics, Melville, New York (This publication).

3. T. W. Koeth et al., "Capture Cavity II at Fermilab," Proceedings of LINAC '06, Knoxville, TN, USA, 2006, pp. 719-721.

4. Buhler, S. et. al, "Status Report of the TTF Capture Cavity Cryostat," in Advances in Cryogenic Engineering 41A, edited by P. Kittel, Plenum Press, New York, 1996, pp. 863-867.

5. DeGraff, B. et al, "SCRF Cryogenic Operating Experience at FNPL," in Advances in Cryogenic Engineering 51B, edited by J. G. Weisend, American Institute of Physics, Melville, New York, 2006, pp. 1164-1171.

6. Harms, E. et al., "Experience with Capture Cavity II," submitted to Proceedings of the 2007 Particle Accelerator Conference, Albuquerque, NM, USA, 2007.

7. Branlard, J. et al., "Capture Cavity II Results at FNAL," submitted to Proceedings of the 2007 Particle Accelerator Conference, Albuquerque, NM, USA, 2007.

8. Hocker, A, private communication, Sept. 5, 2006.

9. McGee, M. and Pischalnikov, Y., "Mechanical Stability Study of Capture Cavity II at Fermilab," submitted to Proceedings of the 2007 Particle Accelerator Conference, Albuqerque, NM, 2007. 